East African Medical Journal Vol. 81 No. 5 May 2004

FEASIBILITY OF MEDICAL MALE CIRCUMCISION IN NYANZA PROVINCE, KENYA

C. L., Mattson, MS., Division of Epidemiology and Biostatistics, School of Public Health, University of Illinois at Chicago, Chicago, IL, R., Muga, MBChB, Ministry of Health, Kenya, R., Poulussen, MD., Flemish Association for Development Cooperation and Technical Assistance, Brussels, Belgium, T., Onyango, Ministry of Health, Kenya and R. C., Bailey, PhD., MPH., Division of Epidemiology and Biostatistics, School of Public Health, University of Illinois Chicago, USA

Request for reprints to: Dr. R. Bailey, Division of Epidemiology and Biostatistics, School of Public Health, University of Illinois Chicago, 1603 W. Taylor St. Chicago, IL 60612, USA

\title{
FEASIBILITY OF MEDICAL MALE CIRCUMCISION IN NYANZA PROVINCE, KENYA
}

\author{
C. L. MATTSON, R. MUGA, R. POULUSSEN, T. ONYANGO and R. C. BAILEY
}

\begin{abstract}
Background: Over forty observational studies have reported a protective effect of male circumcision against HIV-I acquisition, leading some to suggest that male circumcision be added to the limited armamentarium of HIV prevention strategies.

Objectives: To evaluate the feasibility and requirements of implementing medical male circumcision in Nyanza Province of Kenya a quasi-experimental design was implemented to assess clinicians' knowledge of male circumcision and to assess the effectiveness of a one day educational intervention in one district. A cross sectional survey assessed the availability of necessary instruments and surgical supplies.

Results: None of the participating ten health facilities had all the necessary instruments and supplies to safely perform male circumcisions. Though most clinicians reported training about circumcision, most lacked sufficient knowledge to perform the procedure, and few were familiar with the process of informed consent. We demonstrated that a didactic educational workshop significantly improved providers' knowledge of male circumcision.

Conclusions: Since increasing numbers of young men and parents are requesting male circumcision services in many parts of sub-Saharan Africa, health providers must undergo further training in the performance of the procedure and the process of informed consent. Many health facilities will have to be provided with the instruments and supplies necessary to perform male circumcision safely.
\end{abstract}

\section{INTRODUCTION}

By the end of the year 2002, 42 million people were living with HIV/AIDS worldwide and 30 million or $70 \%$ of them were living in sub-Saharan Africa(1). Over $90 \%$ of infections in African adults were transmitted through heterosexual intercourse(1). In the absence of affordable treatment or a vaccine, prevention strategies have become paramount in fighting the pandemic. Current programmes have reported limited success and due to the enormity of the pandemic, other prevention strategies are sorely needed $(2,3)$. One potential new prevention strategy is male circumcision (MC). Approximately 40 observational epidemiologic studies have reported significant associations between lack of MC and HIV-I infection(4). According to recent meta-analyses of 27 studies conducted in sub-Saharan Africa, circumcised men are at half the risk of HIV acquisition compared to uncircumcised men (OR 0.52 [95\% $\mathrm{Cl} 0.40-0.68])(5)$. When adjustment was made for potential confounders, including the number of sex partners, condom use, and ethnicity, an even stronger effect was demonstrated (OR 0.42 95\% Cl 0.34-0.54).

Several hypotheses have been proposed to explain the possible biologic mechanism on how MC confers some protection against HIV acquisition, but the most compelling hypothesis is related to the high density of HIV target cells, namely Langerhans' and CD4 T-cells, in the mucosa of the underside of the foreskin. These cells have been demonstrated to be highly susceptible to HIV- I infection in foreskin tissue in explant culture(6).

The epidemiologic and biologic evidence supporting the increased risk of HIV acquisition through the presence of the foreskin has prompted investigators to encourage consideration of adding MC to the limited armamentarium of strategies to combat the HIV/AIDS in areas where circumcision is not commonly practised, HIV prevalence is high, and transmission is predominantly heterosexual(5,7-9). Despite compelling observational evidence linking HIV acquisition and lack of MC, randomized controlled trials of $\mathrm{MC}$ are needed to definitively conclude that circumcision confers protection without significantly changing risk behaviour (10). Until results from such trials are available and assessed by the international health community, MC should not be actively promoted as an HIV prevention strategy. Meanwhile, however, studies should go forward to assess the feasibility and acceptability of medical MC. In the event that clinical trials of MC demonstrate 
a protective effect against HIV and other sexually transmitted diseases, we should have the information necessary to implement safe and affordable interventions.

In the interests of safety and, in some cases, affordability, many people in societies that practise circumcision as a rite of passage or for religious reasons are increasingly turning from traditional practitioners to clinicians for the procedure. An increasing number of parents in sub-Saharan Africa are having their male children circumcised in local hospitals and clinics. As these trends are likely to continue, it is prudent to determine whether clinics and providers have the necessary resources and training to perform the procedure safely. In addition, mounting evidence suggests that young men and parents in many groups in Africa that do not traditionally practise circumcision are beginning to turn to circumcision for a variety of reasons(12-15). The main factors contributing to the changing attitudes in favour of MC are perceptions of increased cleanliness, reduction in the risk of STD/HIV, sexual pleasure, religion, and ethnic mixing(14).

As medical circumcision gains acceptability among both traditionally circumcising and non-circumcising ethnic groups, information is needed about health care providers' clinical knowledge of the risks and benefits of MC and their ability to perform the procedure. Qualitative studies in Kenya and South Africa have indicated limited knowledge among clinicians of the risks and possible benefits of $\mathrm{MC}$ and a need for targeted training (14). The objectives of this study were first, to assess the providers' procedural and clinical knowledge of MC in two districts in Nyanza Province, second, to assess the effectiveness of a one day educational intervention on providers' knowledge in Siaya District only, and third, to assess the resources available in government health facilities to perform MC in the two districts.

\section{MATERIALS AND METHODS}

This quasi-experimental study, conducted in May - June, 1999, assessed providers' clinical and procedural knowledge of male circumcision in two districts: Siaya district and Nyando district, in Nyanza Province, Kenya, and assessed the effectiveness of the educational intervention on clinicians' knowledge of male circumcision before and after the intervention in Siaya district only. Every eligible (on duty) clinician (i.e. medical officers, clinical officers, and male nurses) in ten general health facilities under the District Medical Office were invited to participate in the study. All participants were fully informed of the risks and benefits of the research and provided informed consent. All study participants completed self-reported questionnaires about their clinical and procedural knowledge of male circumcision and those providers who received the educational intervention also filled out the questionnaire again following the completion of the educational workshop.

The questionnaire was divided into the following sections: demographic characteristics (i.e. age, ethnicity, religion and occupation), circumcision status, knowledge of circumcision as it relates to disease, provider experience with male hygiene and circumcision, and knowledge relating to performing the circumcision procedure itself. The questionnaire was developed in consultation with Dr. J. Otieno, the consulting surgeon for the study. An overall knowledge score was calculated by summing correct answers to the sub-set of questions assessing knowledge of the circumcision procedure and the questions assessing the knowledge of evidence associating circumcision and disease.

The day-long educational intervention was implemented in Siaya district only. The intervention consisted of the following three components: a lecture on existing health risks and benefits of male circumcision; a demonstration of a mock circumcision procedure led by two trained surgeons; and a video of surgical circumcision. This workshop was developed under the guidance of an epidemiologist, two surgeons, a medical officer from the ministry of health, and a member of the district health management team. All available clinicians completed the questionnaire after providing informed consent. In addition, a cross sectional survey of ten clinics, five in each district, were visited by the investigators and an inventory was taken of the surgical supplies and equipment necessary to perform circumcision at each of the ten participating clinics. The checklist was developed with the study consulting surgeon. This study was approved by the Nyanza ethical review board.

Statistical Analyses: Questionnaire responses were entered into QuatroPro then imported and analysed in SPSS Version 10.1 for Windows (Statistical Package for Social Science, SPSS Inc., Chicago). Providers' experience with and knowledge of male circumcision was summarised by generating descriptive statistics for the variables on the questionnaire (i.e. number of circumcisions performed, type of circumcision training, etc.). Comparability between the two districts was assessed by independent $\mathrm{t}$-tests for continuous variables and Pearson Chi-square tests for categorical variables. Because there were no significant differences in provider responses between districts, the descriptive analyses from the two districts are combined. For providers in Siaya district who also completed a questionnaire after the educational intervention, questionnaires before and after the intervention were compared in order to assess if their performance on the knowledge section improved. All continuous variables were compared using paired t-tests and categorical variables were compared by Pearson Chisquare statistics. Test statistics were considered statistically significant if $\mathrm{p}$-values were less than 0.05 . The study had a power of $80 \%$ with an alpha of 0.05 to detect a difference of at least 2.0 in the mean number of correct responses on the knowledge section of the questionnaire.

\section{RESULTS}

Participants: Table I provides the combined sample description of all providers in Siaya and Nyando districts. There were no significant differences between health providers in the two districts. The sample includes 42 providers, 26 in the intervention group and 16 in the control group. All but one of the providers were male. The age of the providers ranged from 2448 years and the mean age was 34 years. Twenty nine $(69 \%)$ of the providers were Luo and the rest were Luhya (16\%), Kalenjin (5\%), Basuba (2\%) and Baganda $(2 \%)$. Of the providers surveyed, $30(71 \%)$ were 
protestants, seven $(17 \%)$ were catholics, two $(5 \%)$ were muslims, and the remaining $5 \%$ identified themselves as having other religious affiliation. The majority of providers were nurses: $23(55 \%), 13(31 \%)$ were clinical officers, three $(7 \%)$ were medical officers, and three $(7 \%)$ were public health or laboratory technicians. Of the 41 male providers, 24 (57\%) were circumcised and $14(33 \%)$ were uncircumcised; four $(10 \%)$ did not report their circumcision status. The median (as well as the mean) age at which men were circumcised was 16 years. Despite only $10 \%$ of Luo men in the general population being circumcised, 12 of the 29 Luo men $(41 \%)$ in this sample reported themselves as circumcised(16). Finally, those in the two districts scored equally on the test score of their knowledge with means of 18.8 out of 28 compared to $19.0(t=0.132$, $\mathrm{p}=0.896)$.

Providers' experience with male hygiene and circumcision: Table 2 presents providers' training and experience with male hygiene and circumcision. There were no differences between districts in the number of circumcisions performed by providers in the last six months. Thirty of 42 providers $(71 \%)$ had performed no circumcision in the six months prior to the survey. Of the eight providers who had performed circumcisions, seven had performed less than four, and one individual performed 20. Eight individuals had performed circumcisions outside of a health facility in their own private practices. Thirty clinicians $(71 \%)$ had received some training on how to perform medical MCs. Of those who received training, $13(43 \%)$ assisted a trained clinician at an actual circumcision procedure, $10(33 \%)$ attended a lecture. Seven (23\%) had performed circumcision with no prior training. The majority $(76 \%)$ of the providers reportedly discussed penile hygiene with at least one patient in the six months prior to the survey. Thirty four $(81 \%)$ reported having discussed male circumcision with patients in the six months prior to the survey, seventeen (41\%) with more than five patients. In addition, there were no differences in providers' experience with hygiene and male circumcision based on providers' circumcision status. One marginal finding was that uncircumcised providers were more likely to discuss penile hygiene with patients than circumcised providers $\left(\chi^{2}=3.14, \mathrm{p}=0.076\right)$. Finally, providers also had similar experiences regarding the number of circumcisions they recommended. Thirty four $(81 \%)$ reported recommending circumcision to at least one patient in the last six months. Nine (21\%) recommended circumcision to more than five patients.

Table 1

Sample description of clinicians in Siaya and Nyando districts in Nyanza province, Kenya $(n=42)$

\begin{tabular}{lc}
\hline Characteristic & Frequency (\%) \\
\hline Median age at time of survey & 33 (range 24-48) \\
Ethnic Group & $29(70)$ \\
$\quad$ Luo & $12(30)$ \\
$\quad$ Non-Luo & $7(18)$ \\
Religion & $30(72)$ \\
$\quad$ Catholic & $4(10)$ \\
$\quad$ Protestant & $3(7)$ \\
Other & $13(31)$ \\
Occupation & $23(55)$ \\
$\quad$ Medical Officer & $3(7)$ \\
Clinical Officer & $24(63)$ \\
$\quad$ Nurse & $14(37)$ \\
Technician & $27(93)$ \\
Circumcision Status & 2 \\
$\quad$ Uncumcised & $(7)$ \\
Circumcision Preference & \\
Circumcised & \\
Uncircumcised & \\
\hline
\end{tabular}


Table 2

Providers' experience with male circumcision $(n=42)$

\begin{tabular}{lc}
\hline Characteristic & Total \\
& No. (\%) \\
\hline Mean circumcisions performed last six months & 1.05 \\
Mean No. circumcisions performed outside clinic & 1.54 \\
Ever trained to perform circumcision & $23(50)$ \\
Yes & $16(40)$ \\
No & $10(33)$ \\
Type of circumcision training & $13(43)$ \\
Lecture only & $7(23)^{*}$ \\
Assisted at circumcision & $11(26)$ \\
Trained while performing circumcision & $9(21)$ \\
No. of patients discussed hygiene last six months & $22(52)$ \\
$0-1$ & $15(36)$ \\
$2-5$ & $9(21)$ \\
More than five & $17(40)$ \\
No. patients discussed circumcision last six months & $18(43)$ \\
$0-1$ & $13(31)$ \\
$2-5$ & $11(26)$ \\
More than five & \\
No. patients recommended circumcision last six months & \\
$0-1$ &
\end{tabular}

*23 clinicians reported having had some formal training; an additional seven reported no training prior to performing their first procedure

Table 3

Providers' correct responses on questionnaire to measure knowledge of male circumcision before and after the educational intervention in Siaya district

\begin{tabular}{|c|c|c|c|c|}
\hline Provider Knowledge & $\begin{array}{l}\text { Pre-Test } \\
\mathrm{n}=26 \\
\text { Mean, s.d. } \\
\% \text { correct }\end{array}$ & $\begin{array}{l}\text { Post-Test } \\
\mathrm{n}=26 \\
\text { Mean, s.d. } \\
\% \text { correct }\end{array}$ & Paired t-test* & p-value \\
\hline Total Knowledge (28 questions) & $\begin{array}{l}19.1,2.7 \\
(68)\end{array}$ & $\begin{array}{l}23.35,2.2 \\
(83)\end{array}$ & -6.84 & $<0.001$ \\
\hline $\begin{array}{l}\text { Knowledge of circumcision } \\
\text { and disease ( } 5 \text { questions) }\end{array}$ & $\begin{array}{l}3.6,0.95 \\
(72)\end{array}$ & $\begin{array}{l}4.31,1.19 \\
(86)\end{array}$ & -3.72 & 0.001 \\
\hline $\begin{array}{l}\text { Procedural Knowledge } \\
\text { ( } 23 \text { questions) }\end{array}$ & $\begin{array}{l}15.5,2.7 \\
(68)\end{array}$ & $\begin{array}{l}19.04,1.77 \\
(83)\end{array}$ & -6.07 & $<0.001$ \\
\hline
\end{tabular}

*d.f. $=25$ for all paired $t$-tests 


\section{Table 4}

Proportion of clinics having working instruments needed for male circumcision $(n=10)$

\begin{tabular}{ll}
\hline Instrument & Total $(\%)$ \\
\hline & \\
Long straight forceps & 40 \\
Mosquito forceps & 20 \\
Needle holding forceps & 60 \\
Dissecting forceps & 20 \\
Suture needles & 30 \\
Kidney dishes & 90 \\
Scissors curved & 30 \\
Waterproof apron & 60 \\
Saucepan with cover & 90 \\
Sterilizer & 70 \\
\hline
\end{tabular}

Providers' knowledge of MC before and after educational intervention: Table 3 shows health care providers' knowledge of male circumcision before and after the educational intervention. After attending the educational intervention, providers' overall knowledge of male circumcision improved by four correct questions out of 28 or increased from $68 \%$ correct to $83 \%$ correct $(\mathrm{t}=-6.84, \mathrm{p}<0.001)$. Twenty four providers' overall score improved from the pre-test to the post-test, one stayed the same, and one did worse on the post-test. The mean score for the sub-set of questions dealing with circumcision as it relates to disease also improved by approximately one question out of five or improved from $72 \%$ to $86 \%$ correct $(p<0.001)$. Finally, there was an increase in the mean correct answers for the sub-set of questions dealing with procedural knowledge of circumcision by approximately 3.5 questions out of 23 or an increase from $68 \%$ to $83 \%$ correct ( $p<0.001$ ). Twenty-one providers improved on this section of the post-test, four stayed the same, and one did worse. In addition, prior to the educational intervention, only 12 $(46 \%)$ of the providers believed that it was necessary to obtain informed consent for the circumcision procedure.

Availability of instruments necessary to perform $M C$ : Table 4 presents a summary of the availability of instruments needed to perform MCs in the participating health clinics. Based on the inventories, it was clear that many clinics were missing the instruments necessary to perform MCs. Seven of the 10 clinics surveyed had the appropriate sterilizers, three of 10 had suture needles, and less than half had forceps necessary to perform circumcisions.

\section{DISCUSSION}

While the international public health community debates the wisdom of promoting MC for HIV prevention in resource poor settings where safety of the procedure may be sub-optimal, many young men and parents are turning to clinical $\mathrm{MC}$ in the belief that circumcision leads to improved hygiene, reduced risk of STDs and
HIV, and less risk of pain and complications than circumcision at the hands of a traditional surgeon (1115). In this study, we attempted to evaluate whether clinicians in government health facilities have the necessary knowledge, surgical training, equipment and supplies to provide safe circumcision services. Previously, we demonstrated that medical professionals in Nyanza Province have limited knowledge about the risks and benefits of male circumcision, and they themselves believe that further training would be necessary if they were to offer circumcision services (14). The results of this study similarly indicate that only approximately half of the health care providers practicing in government health facilities in Nyanza Province have been exposed to any training in $\mathrm{MC}$. Twenty three percent of providers performed their first circumcision with no clinical training in the procedure. Further, our results indicate that after attending a day long educational seminar, procedural and clinical knowledge of male circumcision improved from $67 \%$ up to $83 \%$. Moreover, no facility had the complete kit necessary for the circumcision procedure, and basic expendable supplies such as sutures, anaesthesia and bandages were seldom available. Clearly, if MC is to be made more widely available in Nyanza, resources will have to be allocated to train health providers and to see that health facilities are equipped with the instruments and supplies required for safe procedures.

Several important themes emerge from this study. First, not only are health providers in Nyanza Province unlikely to have the training or supplies necessary to perform medical MC safely, they have little familiarity with the concept of informed consent, by which patients or their guardians should be fully informed of the risks and benefits of the procedure, and be assured of confidentiality. If the ministry of health decides to offer MC services to meet community demands, further training is likely to be necessary. Our data suggest that offering an educational workshop to providers may increase providers' clinical and procedural knowledge regarding MC. However, increased knowledge of MC alone does not constitute sufficient training. Prior to allowing clinicians to perform MC, further hands-on clinical training would be necessary to ensure the health and safety of patients.

In addition, much more extensive information is needed on the rate and nature of complications that may be experienced in various clinical settings. Anecdotes about adverse events occurring in traditional settings abound in the popular press, but there are scant data available from developing country hospitals and clinics as to the frequency of the various complications (17). The published information that exists suggests that complications in African hospitals are more frequent than in western countries (17), but that the great majority are mild (eg, post-operative bleeding and infection) and quite easily resolved. Any interventions will have to concentrate on providing the training and 
facilities necessary to minimise adverse events and to address them correctly should they occur.

Further, there is one randomized controlled trial of male circumcision and HIV-1 acquisition currently underway in Kisumu, Kenya, and two additional trials being conducted in Uganda and South Africa. These trials will take three to five years to complete. If the trials demonstrate efficacy, the feasibility and operational requirements of introducing $\mathrm{MC}$ must be known. This study indicates that introducing $\mathrm{MC}$ would require extensive provider training as well as resources for upgrading clinics with the necessary equipment and supplies. As estimates of the protective effect of MC against HIV and other sexually transmitted infections become available, further assessment of operational requirements for MC interventions will be useful to permit calculations of costs and cost effectiveness of MC interventions.

\section{ACKNOWLEDGEMENTS}

To A. Ogollah, E. Ogonji, and M. Orao for their assistance with recruitment of participants and collection of data, to Dr. J. Otieno who assisted with training of clinicians, to an anonymous referee for helpful comments, and to the clinicians and residents of Siaya and Nyando districts for their generous participation in this study. The design and conduct of this study were approved by the Nyanza Ethical Review Board.

\section{REFERENCES}

1. Joint United Nations Programme on HIV/AIDS(UNAIDS) and World Health Organization (WHO). Report on the Global HIV/AIDS Epidemic, Geneva: UNAIDS, 2001.

2. Ngugi, E., Wilson, D., Sebstad, P., Plummer, F., and Moses, S., Focused peer-mediated educational programs among female sex workers to reduce sexually transmitted disease and human immunodeficiency virus transmission in Kenya and Zimbabwe. J. Infectious Dis. 1996; 74:(S2) 40-47.

3. Asimmwe, O. G., Opio, A., Musinguzi, J., Madraa, E., Tebmo, G., and Careal, M. Change in sexual behavior and decline in HIV infection among young pregnant women in urban Uganda. AIDS; 1997; 11:1757-1763.
4. Bailey, R. C., Plummer, F. A., and Moses, S., Male circumcision and HIV prevention: current knowledge and future research directions. The Lancet Infec. Dis. 2001; 1:223-231.

5. Weiss, H.A., Quigley, M.A., and Hayes, R. J., Male circumcision and risk of HIV infection in sub-Saharan Africa; a systematic review and meta-analysis. AIDS; 2000; 14:2261-2370.

6. Patterson, B. K., Landay, A., Siegel, J. N., Z. Flener, D., Pessis, A., Chaviano, and R. C., Bailey. Susceptibility to human immunodeficiency virus- I infection of human foreskin and cervical tissue grown in explant culture. Amer. J. Pathology; 2002; 161:867-873.

7. Halperin, D. T., and Bailey, R. C. Male circumcision and HIV infection: 10 years and counting. Lancet 1999; 354: 1813-1815.

8. Buve, A., B., Auvert, E., Lagarde, M., Kahindo, Hayes, R., and Carael, M. Male circumcision and HIV spread in sub-Saharan Africa. Abstracts, XIII International Conference on AIDS (MoOrC192), Durban, South Africa.

9. Moench, T. R., Neglected opportunities. J. Infec. Dis. 2000; 181: 804-805.

10. van Dam, J., and Anastasi, M. C., Male Circumcision and HIV prevention. Population Council, 2000.

11. Caldwell, J. C., and Caldwell, P., The African AIDS epidemic. Sci. Amer. 1996; 274:62-68.

12. Nnko, S., R., Washija, Urassa, M., and Boerma, J. T., Dynamics of male circumcision practices in Northwest Tanzania. Sexually Trans. Dis. 2001; 28:214-218.

13. Mattson, C. M., Bailey, R. C., Muga, R., and Poulussen, R., Attitudes and beliefs about male circumcision and predictors of circumcision preference in Nyanza Province, Kenya. AIDS Care (in press).

14. Bailey, R.C., Muga, R., Poulussen, R. and Abicht H. The acceptability of male circumcision to reduce HIV infections in Nyanza Province, Kenya. AIDS Care, 2002; 14:27-40.

15. Taljaard, R., Taljaard, D., Auvert, B., and Neilssen, G., Cutting it fine: male circumcision practices and the transmission of STDs in Carletonville. Abstracts, XIII International conference on AIDS (MoOrC 195), Durban, South Africa.

16. Auvert, B., Buve, A., Lagarde, E., et al. Study group on the heterogeneity of HIV epidemics in African cities. Male circumcision and HIV infection in four cities in subSaharan Africa. AIDS; 2001; 15 (suppl 3): S31-30.

17. Magoha, G. A., Circumcision in various Nigerian and Kenyan hospitals. East Afr. Med. J. 1999; 76:583-586. 